Article

www.mdpi.com/journal/polymers

\title{
Poly(butylene succinate) Ionomers with Enhanced Hydrodegradability
}

\section{Mayka Bautista, Antxon Martínez de Ilarduya *, Abdelilah Alla and \\ Sebastián Muñoz-Guerra *}

Departament d'Enginyeria Química, Universitat Politècnica de Catalunya, Diagonal 647, Barcelona 08028, Spain; E-Mails: maykairina@hotmail.com (M.B.); abdel.alla@upc.edu (A.A.)

* Authors to whom correspondence should be addressed;

E-Mails: antxon.martinez.de.ilarduia@upc.edu (A.M.I.); sebastian.munoz@upc.edu (S.M.-G.); Tel.: +34-9340-10910 (A.M.I.); +34-9340-16680 (S.M.-G.);

Fax: +34-9340-17150 (A.M.I.); +34-9340-17150 (S.M.-G.).

Academic Editor: Christine Wandrey

Received: 7 May 2015 / Accepted: 30 June 2015 /Published: 9 July 2015

\begin{abstract}
A series of poly(butylene succinate) (PBS) ionomers containing up to $14 \mathrm{~mol} \%$ of sulfonated succinate units have been synthesized by polycondensation in the melt-phase. The copolyesters were obtained with weight average molecular weights oscillating between 33,000 and $72,000 \mathrm{~g} \cdot \mathrm{mol}^{-1}$. All copolyesters were semicrystalline with melting temperatures and enthalpies decreasing and glass transition temperatures increasing with the content of ionic units. The thermal stability of PBS was slightly reduced by the incorporation of these units, and it was also found that the copolyesters were stiffer but also more brittle than PBS. The hydrolytic degradability of PBS was enhanced by copolymerization, an effect that was much more pronounced in basic media.
\end{abstract}

Keywords: PBS copolyesters; ionomers; thermal properties; hydrolytic degradation; sulfonated dimethyl succinate

\section{Introduction}

The electrostatic effect exerted by the ionic groups on the packing of polymer chains strengthens the intermolecular forces and leads to physical cross-linking. Eisenberg and Rinaudo [1] proposed that 
the bulk properties of ionomers are governed by ionic interactions taking place in discrete regions of the material. In the recent decades, ionomers have been extensively investigated to examine their unique characteristic ionic aggregation as responsible for significant changes in physical properties such as glass-transition temperature, mechanical behavior, transport of gases, and melt viscosity [2-6]. According to the Eisenberg-Hird-Moore model, at increasing ionic concentrations, the aggregates (multiplets) start to overlap to form the so-called clusters $[7,8]$.

Biodegradable polymers are considered ideal materials for environmental protection and they are also of great interest for biomedical applications. Particular attention has been given to aliphatic polyesters due to their relatively easy synthesis, good biocompatibility and biodegradability, and acceptable overall pattern of properties [9-13]. Among them, poly(butylene succinate) (PBS) is nowadays one of the most appealing biodegradable polymer because it is fully sustainable, commercially available and exhibits a good balance of thermal and mechanical properties. Nevertheless, a good amount of work still remains to be done on this polyester in order to optimize its synthesis and to attain the level of performance required by current advanced applications.

Copolymerization is one of the most important methods for modifying the physical properties of polyesters. However, the random incorporation of a second monomeric unit unavoidably entails a significant depression in the melting temperature of crystalline polymers that may restrict the temperature range over which the copolyester can be used to unacceptable values [14-17]. In order to surpass this shortcoming, some authors have introduced the ionomer approach into the field of bio-based polyesters $[18,19]$. Han et al. $[9,20]$ inserted ionic groups into the PBS main chain leading to an improved extensibility due to reduction of crystallinity as well as a noticeable modification in melt rheological properties due to the ionic interactions. PBS ionomers have been proposed as a solution for the brittleness and poor processability of other polymers. Park et al. [21] blended polylactic acid (PLA) with PBS ionomers with the aim of improving the shortcomings of PLA.

The aim of this work is to report on the effect of sodium 1,2-(dimethoxycarbonyl)ethanesulfonate as a comonomer on the properties of poly(butylene succinate). This comonomer is dimethyl succinate bearing a pendant sodium sulfonate group, which makes the compound ionic and non-symmetric. The presence of this unit is expected to modify the properties of the polyester without disturbing its packing in the solid state much since its differences in size with the succinate unit are relatively small. Two papers have previously reported on these type of copolyesters, both of them dealing with very low content of ionic groups and focused exclusively on crystallization aspects [22,23]. In the present work, we have prepared a series of PBS copolyesters, abbreviated as $\mathrm{PBS}_{x} \mathrm{SS}_{y}$, containing sulfonated succinic units (SS) in a range much wider than previously reported, i.e., from 3 to $15 \mathrm{~mol} \%$ and repressing the elimination of sodium sulfonate groups during reaction. The object is to evaluate how the basic properties of PBS and, in particular, hydrolytic degradability are affected by the incorporation of sulfonated succinate units, with special attention to copolyesters with high content in ionic groups. 


\section{Experimental Section}

\subsection{Materials}

Succinic acid (SA) (99\%) was purchased from Panreac Química S.L.U. (Barcelona, Spain). 1,4-Butanediol (99\%) and the catalyst dibutyl tin oxide (DBTO, 98\%), dimethyl fumarate (DMF) and sodium bisulfite were purchased from Sigma-Aldrich (Madrid, Spain). Solvents used for purification and characterization such as diethyl ether, dimethyl sulfoxide, chloroform, trifluoroacetic acid, methanol and dichloroacetic acid were purchased from Panreac Química S.L.U. (Barcelona, Spain). All the reagents and solvents were of either technical or high-purity grade and were used as received without further purification.

\subsection{Analytical Techniques}

Intrinsic viscosities of the copolyester dissolved in dichloroacetic acid were measured with an Ubbelohde viscometer thermostated at $25 \pm 0.1{ }^{\circ} \mathrm{C}$. Size exclusion chromatography (SEC) was performed on a Waters system (Waters Corporation: Milford, MA, USA) equipped with a refractive index detector (RID-10A) using 1,1,1,3,3,3-hexafluoro-2-propanol (HFIP) as the mobile phase. Molecular weights and their distribution were calculated against poly(methyl methacrylate) standards using the Millenium 820 software (Waters Corporation: Milford, MA, USA). To prevent ionic aggregation, polymer samples were previously dissolved in a mixture of chloroform/trifluoroacetic acid (1/1) and precipitated with methanol except those containing 10 and $15 \mathrm{~mol} \%$ of ionic units that were precipitated with diethyl ether.

NMR spectra were recorded on a Bruker AMX-300 spectrometer (Bruker Corporation, Wissembourg, France) operating at $300.1 \mathrm{MHz}$ for ${ }^{1} \mathrm{H}$ and $75.5 \mathrm{MHz}$ for ${ }^{13} \mathrm{C}$. About $10 \mathrm{mg}$ for ${ }^{1} \mathrm{H}$ or $50 \mathrm{mg}$ for ${ }^{13} \mathrm{C}$ of polymer samples were dissolved in a mixture $(70 / 30, v / v)$ of deuterated chloroform $\left(\mathrm{CDCl}_{3}\right)$ /trifluoroacetic acid (TFA). Sixty four and 5000-10,000 scans were acquired with 32,768 (32 k) and 65,336 (64 k) data points, and 1 and $2 \mathrm{~s}$ of relaxation delays for ${ }^{1} \mathrm{H}$ and ${ }^{13} \mathrm{C}$, respectively. The thermal behavior of the polyesters was examined by differential scanning calorimetry (DSC) with a PerkinElmer DSC Pyris 1 instrument (Madrid, Spain) calibrated with indium and zinc for the temperature and enthalpy. DSC data were obtained from 4 to $6 \mathrm{mg}$ samples at heating and cooling rates of $10{ }^{\circ} \mathrm{C} \cdot \mathrm{min}^{-1}$ under nitrogen circulation $\left(20 \mathrm{~mL} \cdot \mathrm{min}^{-1}\right)$. The $T_{\mathrm{g}}$ of polyesters was measured from amorphous samples at a heating rate of $20{ }^{\circ} \mathrm{C} \cdot \mathrm{min}^{-1}$. Thermogravimetic analysis (TGA) measurements were carried out with 10 to $15 \mathrm{mg}$ of sample under a nitrogen flow of $20 \mathrm{~mL} \cdot \mathrm{min}^{-1}$ at a heating rate of $10{ }^{\circ} \mathrm{C} \cdot \mathrm{min}^{-1}$ and within a temperature range of $30-600^{\circ} \mathrm{C}$, using a Perkin-Elmer TGA6 thermobalance (Madrid, Spain).

Tensile testing was performed on bone shape specimens $\left(2.7 \times 10 \mathrm{~mm}^{2}\right)$ which were cut from isotropic films obtained by hot pressing with a thickness of about $200 \mu \mathrm{m}$. Tensile tests were conducted at room temperature on a Zwick BZ2.5/TN1S (Ulm-Einsingen, Germany) universal tensile testing apparatus operating at a constant crosshead speed of $10 \mathrm{~mm} \cdot \mathrm{min}^{-1}$ with a $0.5 \mathrm{~N}$ preload and a grip-to-grip separation of $20 \mathrm{~mm}$. Five measurements were taken for each copolyester and the results are reported as average values. 


\subsection{Synthesis of Sulfonated Dimethyl Succinate}

Sodium 1,2-(dimethoxycarbonyl)ethanesulfonate (sulfonated dimethyl succinate) (SDMS) was synthesized according to the procedure described elsewhere [24]. Dimethyl fumarate $(5 \mathrm{~g})$ and $\mathrm{NaHSO}_{3}(8 \mathrm{~g})$ were dissolved in a methanol/water solution $(70 / 30, v / v)$ and were left under reflux at $80{ }^{\circ} \mathrm{C}$ for $8 \mathrm{~h}$. Then, the reaction mixture was evaporated to dryness. The residue was extracted with DMSO, the liquid phase was concentrated and precipitated with a large amount of acetone, and the precipitate collected by filtration and dried under vacuum at $60{ }^{\circ} \mathrm{C}$ for $48 \mathrm{~h}$. The structure of the SDMS comonomer was checked by NMR; both ${ }^{1} \mathrm{H}$ and ${ }^{13} \mathrm{C}$ NMR spectra with signals assignments are available in the electronic supplementary information file (Figure S1).

\subsection{Polymers Synthesis}

PBS and poly(butylene succinate-co-butylene sulfonated succinate) ( $\mathrm{PBS}_{x} \mathrm{SS}_{y}$ ) copolyesters were prepared according to the synthetic route depicted in Scheme 1. It consisted of a two-step melt polycondensation procedure corresponding to esterification/transesterification and polycondensation reactions, respectively. A mixture SA, BD and SDMS with the selected composition and containing $0.6 \%(w / w)$ of dibutyl tin oxide (DBTO) was charged into a $250 \mathrm{~mL}$, three-necked, round-bottom flask equipped with a mechanical stirrer, a nitrogen inlet, and a distillation column. A molar ratio 2.1:1 of BD to SA or SA + SDMS was used for both PBS and $\mathrm{PBS}_{x} \mathrm{SS}_{y}$ copolyesters. The esterification/transesterification reaction was carried out at $170{ }^{\circ} \mathrm{C}$ under a nitrogen flow for a period of $6 \mathrm{~h}$ with continuous removal of the released water and methanol. Polycondensation reaction leading to PBS homopolymers was performed either at 240 or $180^{\circ} \mathrm{C}$ for $3 \mathrm{~h}$ under a $0.03-0.006$ mbar vacuum using DBTO as a catalyst. Two PBS samples differing in molecular weight (PBS1 and PBS2) were respectively obtained. Polycondensation reactions leading to $\mathrm{PBS}_{x} \mathrm{SS}_{y}$ copolyesters were left to proceed at $190{ }^{\circ} \mathrm{C}$ under high vacuum for 6-10 h depending on composition. The high viscous liquid finally formed was cooled down to room temperature and the atmospheric pressure was restored with a nitrogen flow to prevent degradation. If it is not specified, the final solid mass without further treatment was used for characterization and property evaluation.

\subsection{Hydrolytic Degradation}

The hydrolytic degradation essays for PBS and $\mathrm{PBS}_{x} \mathrm{SS}_{y}$ samples were carried out in parallel using three different buffer solutions: citric acid ( $\mathrm{pH} 4.0)$, sodium phosphate $(\mathrm{pH} 7.4)$ and sodium carbonate buffer $\left(\mathrm{pH} \mathrm{10)}\right.$ at $37{ }^{\circ} \mathrm{C}$. The films were prepared by hot pressing at a temperature just below the melting point of the polymer. They were cut into $7 \times 7 \times 0.20 \mathrm{~mm}$ pieces. Samples were immersed in $10 \mathrm{~mL}$ of each buffer for 8 weeks. After a fixed period of time the samples were picked out and washed with distilled water and dried in a vacuum oven at room temperature to constant weight and finally analyzed by Gel Permeation Chromatography (GPC). For NMR spectroscopy analysis samples of about $30 \mathrm{mg}$ weight were immersed in NMR tubes containing the corresponding buffer in $\mathrm{D}_{2} \mathrm{O}$, and incubated under the same conditions. Both the products released to the incubating medium as well as the residue left after incubation were subjected to NMR analysis. 


\section{Results and Discussion}

\subsection{Synthesis and Chemical Structure of PBSxSS Copolyesters}

The synthesis pathway leading to the $\mathrm{PBS}_{x} \mathrm{SS}_{y}$ ionomers is shown in Scheme 1. The procedure consists of two successive steps, the first one is an esterification/transesterification reaction leading to low molecular weight oligomers, and the second one is the polycondensation of the oligomers formed in the previous step to render the final copolyesters. The first step was carried out at $170{ }^{\circ} \mathrm{C}$ with removal of the released water and methanol and the second one was carried out at higher temperatures under high vacuum to accelerate the transesterification reaction and to unbalance the equilibrium towards the formation of high molecular weight polymers by releasing of the excess of BD. The maximum temperature applied was in all cases $190{ }^{\circ} \mathrm{C}$ because the $\beta$-elimination reaction of SS units with releasing of $\mathrm{NaHSO}_{3}$ and concomitant generation of fumarate units started to happen above this temperature.

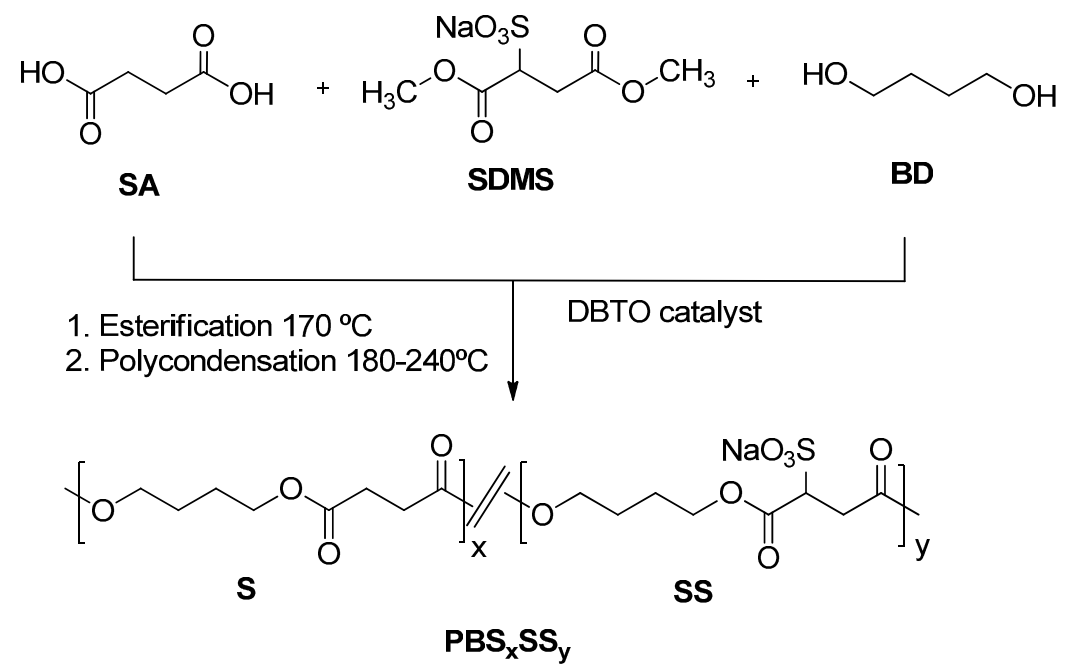

Scheme 1. Polymerization reactions leading to homopolyesters and $\mathrm{PBS}_{x} \mathrm{SS}_{y}$ copolyesters.

It was observed that the melt viscosity attained by the reaction mass increased gradually with the content of SS units as it should be expected for ionomers; it is widely known that sulfonate groups can interact to generate ionic aggregates or clusters which act as thermoreversible crosslinks [25,26]. Dibutyl tin oxide (DBTO) was the catalyst of choice. Initially, the synthesis of the copolymers was done with titanium (IV) tetrabutoxide (TBT), but the result was not satisfactory due to the decomposition of SS units and formation of copolyesters with very low intrinsic viscosities. DBTO allowed the reaction to proceed at lower temperatures and copolymers with significantly higher intrinsic viscosities could be then attained. All the copolyesters were obtained in high yields (higher than 90\%) and molecular weights fell with increasing content in SS units (Table 1). It should be noted that GPC measurements could be affected by ionic aggregations. Although one can expect that the treatment of samples with TFA previous to injection and the use of HFIP as the mobile phase will minimize the occurrence of ionic interactions, their complete absence cannot be fully ascertained. However results afforded by GPC were very consistent with intrinsic viscosities measured in dichloroacetic acid (DCA), which ranged between 0.74 and $1.18 \mathrm{dL} \cdot \mathrm{g}^{-1}$ with values also decreasing as 
the content of the copolyester in SS units increased. Nevertheless, the clear conclusion that can be drawn from these data is that the polycondensation reaction is hindered in the presence of the dimethyl sulfonated succinate comonomer. One factor motivating the decrease in molecular weight is the limited temperature at which polycondensation has to be conducted in order to avoid the $\beta$-elimination reaction of sulfonate groups. A second factor is the increase in melt viscosity of the mass reaction with the content of the forming copolyester in SS units, which hinders the volatilization of BD that is released in the polycondensation step.

Table 1. Composition and molecular weights of poly(butylene succinate) (PBS) homopolyesters and poly(butylene succinate-co-butylene sulfonated succinate) $\left(\mathrm{PBS}_{x} \mathrm{SS}_{y}\right)$ copolyesters.

\begin{tabular}{|c|c|c|c|c|c|}
\hline \multirow{2}{*}{ Copolyester } & \multirow{2}{*}{$\begin{array}{c}\text { Copolyester composition }^{\text {a }} \\
\text { S/SS }\end{array}$} & \multirow{2}{*}{$\begin{array}{c}\text { Copolyester composition }^{\text {b }} \\
\text { S/SS }\end{array}$} & \multicolumn{3}{|c|}{ Molecular weights } \\
\hline & & & {$[\eta]^{\mathrm{c}}$} & $M_{\mathrm{w}} \mathrm{d}$ & $\boldsymbol{D}^{\mathrm{d}}$ \\
\hline PBS1 ${ }^{\mathrm{e}}$ & $100 / 0$ & $100 / 0$ & 1.33 & 112,000 & 2.2 \\
\hline $\operatorname{PBS} 2^{\mathrm{f}}$ & $100 / 0$ & $100 / 0$ & 1.0 & 45,600 & 2.5 \\
\hline $\mathrm{PBS}_{97} \mathrm{SS}_{3}$ & $97 / 3$ & $97.9 / 2.1$ & 1.18 & 72,000 & 2.5 \\
\hline $\mathrm{PBS}_{95} \mathrm{SS}_{5}$ & $95 / 5$ & $96.1 / 3.9$ & 1.03 & 39,000 & 2.4 \\
\hline $\mathrm{PBS}_{92} \mathrm{SS}_{8}$ & $92 / 8$ & $92.8 / 7.2$ & 1.02 & 38,000 & 2.4 \\
\hline $\mathrm{PBS}_{90} \mathrm{SS}_{10}$ & $90 / 10$ & $90.9 / 9.1$ & 0.94 & 35,000 & 2.2 \\
\hline $\mathrm{PBS}_{85} \mathrm{SS}_{15}$ & $85 / 15$ & $86.2 / 13.8$ & 0.74 & 33,000 & 2.1 \\
\hline
\end{tabular}

${ }^{a}$ Molar ratio of comonomers in the initial feed; ${ }^{b}$ Composition of the copolyester ( $\mathrm{mol} / \mathrm{mol}$ ) determined by ${ }^{1} \mathrm{H}$ NMR;

${ }^{\mathrm{c}}$ Intrinsic viscosity $\left(\mathrm{dL} \cdot \mathrm{g}^{-1}\right)$ measured in dichloroacetic acid at $25^{\circ} \mathrm{C} ;{ }^{\mathrm{d}}$ Weight-average molecular weight $\left(M_{\mathrm{w}}\right)\left(\mathrm{g} \cdot \mathrm{mol}^{-1}\right)$ and dispersity $(\nexists)$ determined by GPC; ${ }^{\mathrm{e}}$ Sample prepared at a polycondensation temperature of $240{ }^{\circ} \mathrm{C} ;{ }^{\text {f }}$ Sample prepared at a polycondensation temperature of $180{ }^{\circ} \mathrm{C}$.
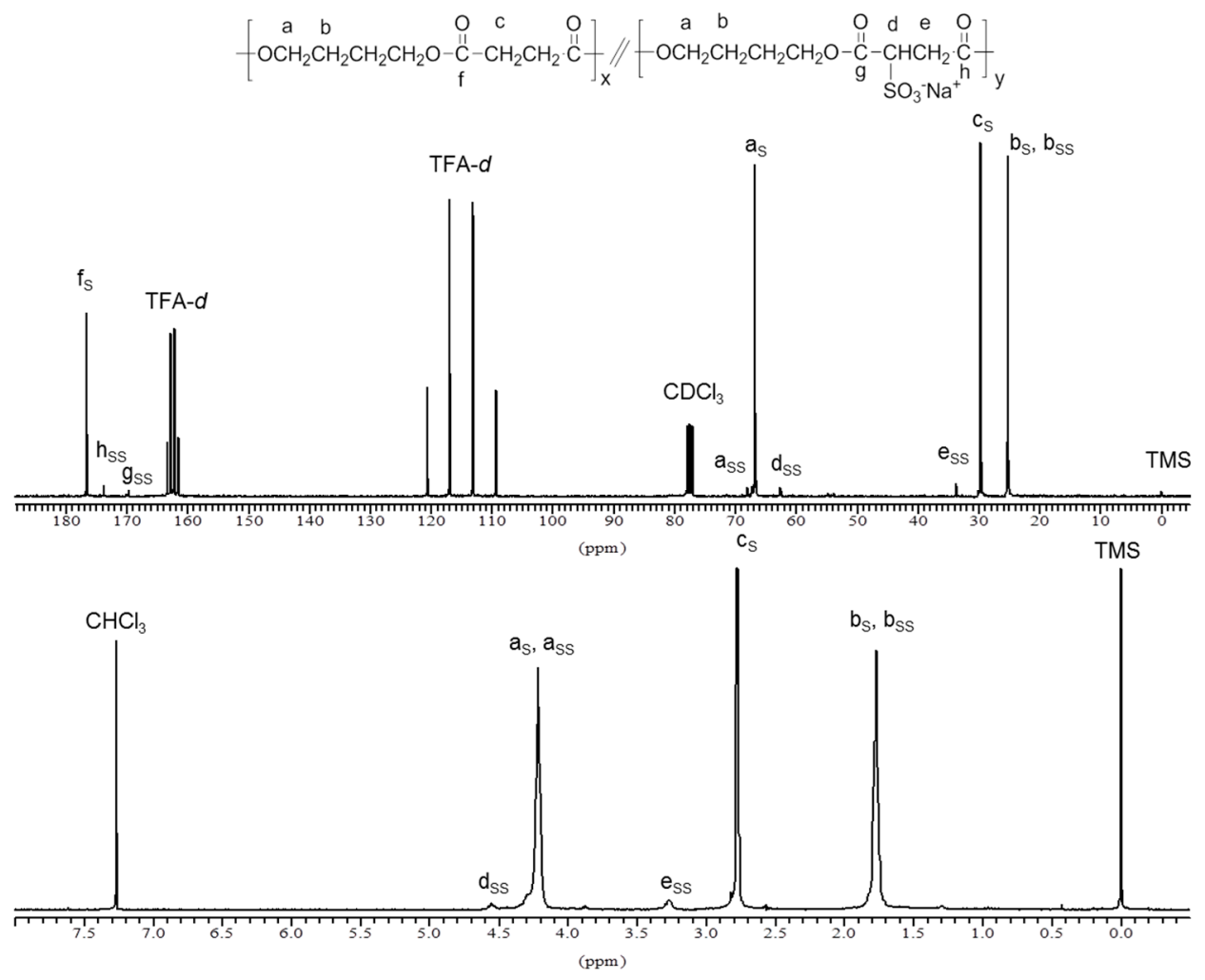

Figure 1. ${ }^{1} \mathrm{H}$ NMR (bottom) and ${ }^{13} \mathrm{C}$ NMR (top) of $\mathrm{PBS}_{85} \mathrm{SS}_{15}$ copolyester. 
The chemical structure and composition of $\mathrm{PBS}_{x} \mathrm{SS}_{y}$ copolyesters was ascertained by NMR. The ${ }^{1} \mathrm{H}$ and ${ }^{13} \mathrm{C}$ NMR spectra of $\mathrm{PBS}_{85} \mathrm{SS}_{15}$ are shown in Figure 1 as representative of the series. The content in SS units was determined by the ratio of the integrated NMR signals at $3.25 \mathrm{ppm}$ for the $\mathrm{CH}_{2}$ in the SS unit (signal e) and at 2.76 ppm for the two $\mathrm{CH}_{2}$ in the succinate unit (signal c). Results are listed in Table 1 indicating that the content of copolyesters in sulfonated units was lower than in the feed. Differences are however small, around $10 \%$ in overall, and they must be attributed to the $\beta$-elimination reaction of sulfonated groups happening in the SS units. This side reaction was observed to be enhanced at higher temperatures [22]; as it is shown in the supplementary information file (Figure S2), the peak corresponding to fumarate protons becomes clearly observed in the ${ }^{1} \mathrm{H}$ NMR spectra of a copolyester heated at $210^{\circ} \mathrm{C}$ for $30 \mathrm{~min}$.

\subsection{Thermal and Mechanical Properties}

The effects exerted by the incorporation of SS units on the thermal properties of PBS were evaluated by DSC and TGA. The DSC data obtained for the whole $\mathrm{PBS}_{x} \mathrm{SS}_{y}$ series examined in this work are collected in Table 2 . The two samples of PBS showed practically the same $T_{\mathrm{g}}$ value of $-37^{\circ} \mathrm{C}$, which was found to increase up to $-32{ }^{\circ} \mathrm{C}$ with the content in SS units. Such slight but significant increase in $T_{\mathrm{g}}$ is supposedly caused by the ionic interactions taking place between the sulfonate groups, which are known to act as physical crosslinks that restrict the chain mobility. Eisenberg et al. [27,28], reported a similar behavior for styrene-based ionomers, which contain ionic multiplets, each of them surrounded by a shell of restricted chain mobility. The restricted mobility region surrounding an isolated multiplet would be too small as to have a noticeable effect on the overall $T_{\mathrm{g}}$ of the polymer, but the multiplet itself would increase the $T_{\mathrm{g}}$ of the polymer by acting as a large cross-link. A similar effect has been reported by Im et al. [22,23] for the same copolyesters with low content of SS units and attributed to the restricted chain mobility caused by the strong intermolecular association of ionic groups.

To evaluate the thermal stability of the polyesters, TGA measurements were carried out under a nitrogen atmosphere in the $30-600{ }^{\circ} \mathrm{C}$ interval. The TGA traces of $\mathrm{PBS}_{x} \mathrm{SS}_{y}$ copolyesters are shown in Figure 2a where the trace for PBS1 has been included for comparison, and data obtained therefrom are summarized in Table 2, which shows that the thermal stability of copolyesters decreased with the content in SS units. Thermal decomposition of PBS occurs in a single stage with maximum rate at about $405{ }^{\circ} \mathrm{C}$ with a $1 \%-2 \%$ of residual weight left upon heating at $600{ }^{\circ} \mathrm{C}$. The insertion of SS units in PBS not only tends to decrease the decomposition temperature but also makes the process more complex. $\mathrm{Up}$ to four decomposition steps are detected in the $\mathrm{PBS}_{x} \mathrm{SS}_{y}$ copolyesters with that happening at 390-400 ${ }^{\circ} \mathrm{C}$ being much more prominent than the others (Figure 2b). The remaining weight after the thermal degradation at $600{ }^{\circ} \mathrm{C}$ increased continuously with the content in SS units, which may be accounted for by the metallic residue left in the decomposition of the SS units (Table 2). 

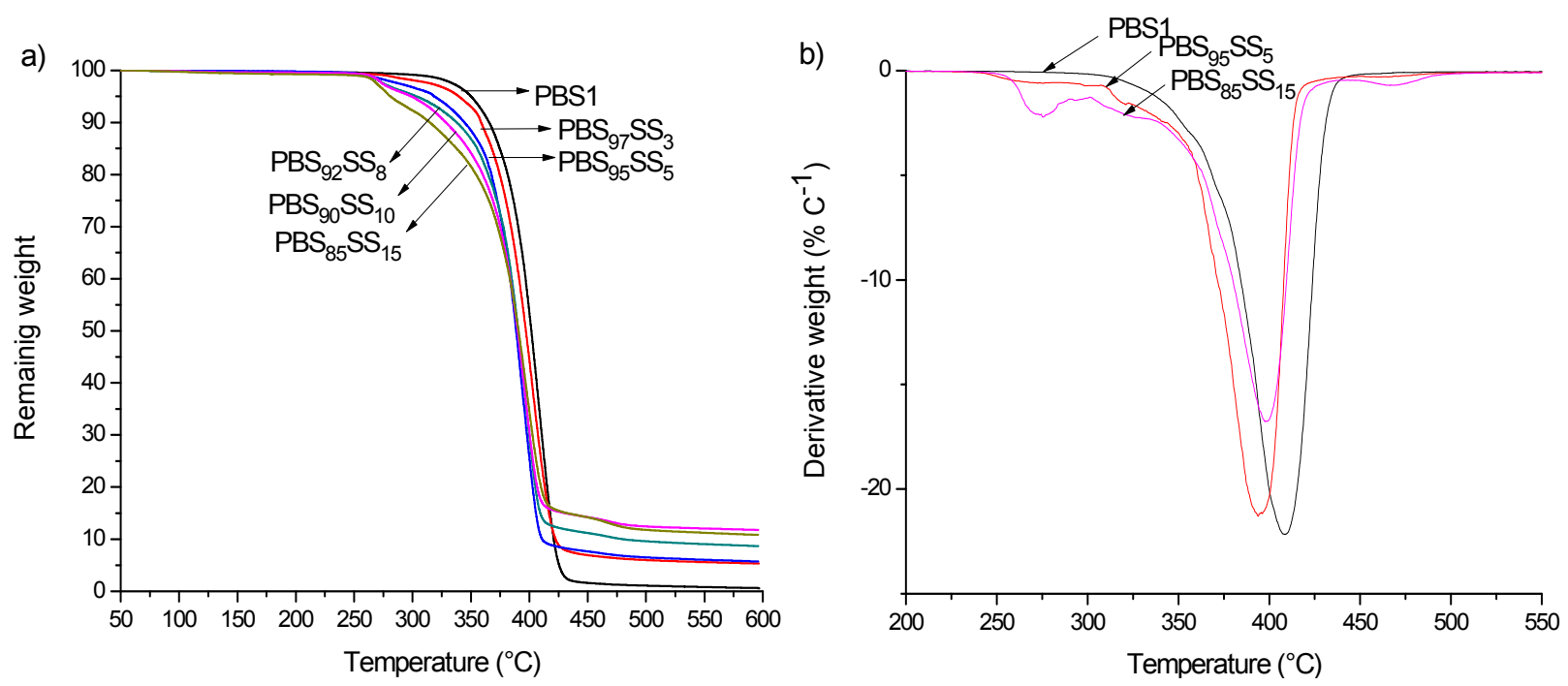

Figure 2. (a) TGA traces of PBS1 and $\mathrm{PBS}_{x} \mathrm{SS}_{y}$; (b) Derivative curves of PBS1 and a selection of $\mathrm{PBS}_{x} \mathrm{SS}_{y}$ copolyesters.

Table 2. Thermal and mechanical properties of PBS homopolymer and $\mathrm{PBS}_{x} \mathrm{SS}_{y}$ copolyesters.

\begin{tabular}{|c|c|c|c|c|c|c|c|c|c|c|c|}
\hline \multirow[b]{2}{*}{ Copolyester } & \multicolumn{3}{|c|}{ TGA } & \multicolumn{5}{|c|}{ DSC } & \multicolumn{3}{|c|}{ Stress-strain Parameters } \\
\hline & $\begin{array}{l}T_{\mathrm{d}}{ }^{\mathrm{a}} \\
\left({ }^{\circ} \mathrm{C}\right)\end{array}$ & $\begin{array}{c}{ }^{\max } T_{\mathrm{d}}{ }^{b} \\
\left({ }^{\circ} \mathrm{C}\right) \\
\end{array}$ & $\begin{array}{c}R W^{\mathrm{c}} \\
(\%)\end{array}$ & $\begin{array}{l}T_{\mathrm{g}}{ }^{\mathrm{d}} \\
\left({ }^{\circ} \mathrm{C}\right)\end{array}$ & $\begin{array}{l}T_{\mathrm{m}}{ }^{\mathrm{e}} \\
\left({ }^{\circ} \mathrm{C}\right)\end{array}$ & $\begin{array}{l}\Delta H_{\mathrm{m}}{ }^{\mathrm{e}} \\
\left(\mathrm{J} \mathrm{g}^{-1}\right)\end{array}$ & $\begin{array}{l}T_{\mathbf{c}}{ }^{\mathrm{f}} \\
\left({ }^{\circ} \mathbf{C}\right)\end{array}$ & $\begin{array}{c}\Delta H_{\mathrm{c}}{ }^{\mathrm{f}} \\
\left(\mathrm{J} \mathrm{g}^{-1}\right)\end{array}$ & $\begin{array}{c}E^{\mathrm{g}} \\
\text { (Mpa) }\end{array}$ & $\begin{array}{l}\sigma_{\max }{ }^{h} \\
(\mathrm{Mpa})\end{array}$ & $\begin{array}{c}\max i \\
(\%)\end{array}$ \\
\hline PBS1 & 363 & 408 & 1 & -37 & $115(114)$ & $70(74)$ & 75 & 66 & $440 \pm 5$ & $35 \pm 1$ & $282 \pm 10$ \\
\hline PBS2 & 356 & 405 & 3 & -37 & $113(114)$ & $77(67)$ & 78 & 62 & $403 \pm 3$ & $26 \pm 1$ & $10 \pm 1$ \\
\hline $\mathrm{PBS}_{97} \mathrm{SS}_{3}$ & 359 & 403 & 5 & -36 & $113(111)$ & $90(69)$ & 71 & 65 & $480 \pm 10$ & $36 \pm 2$ & $121 \pm 3$ \\
\hline $\mathrm{PBS}_{95} \mathrm{SS}_{5}$ & 344 & 399 & 6 & -36 & $113(111)$ & $71(66)$ & 63 & 63 & $515 \pm 10$ & $39 \pm 1$ & $21 \pm 3$ \\
\hline $\mathrm{PBS}_{92} \mathrm{SS}_{8}$ & 338 & 397 & 9 & -34 & $111(110)$ & $61(60)$ & 54 & 57 & $860 \pm 5$ & $42 \pm 1$ & $9 \pm 1$ \\
\hline $\mathrm{PBS}_{90} \mathrm{SS}_{10}$ & 328 & 396 & 12 & -33 & $110(108)$ & $43(56)$ & 53 & 50 & $930 \pm 7$ & $38 \pm 1$ & $6 \pm 1$ \\
\hline $\mathrm{PBS}_{85} \mathrm{SS}_{15}$ & 315 & 393 & 13 & -32 & $106(105)$ & $53(46)$ & 36 & 25 & $1050 \pm 8$ & $28 \pm 2$ & $3 \pm 1$ \\
\hline
\end{tabular}

${ }^{\text {a }}$ Degradation temperature at which a $10 \%$ weight loss was observed in TGA traces at $10^{\circ} \mathrm{C} \cdot \mathrm{min}^{-1}$; ${ }^{\mathrm{b}}$ Temperature of maximum degradation rate for the main decomposition step; ${ }^{\mathrm{c}}$ Remaining weight at $600{ }^{\circ} \mathrm{C} ;{ }^{\mathrm{d}}$ Glass transition temperature taken as the inflection point of the heating DSC traces of melt-quenched samples recorded at $20^{\circ} \mathrm{C} \cdot \mathrm{min}^{-1}$; ${ }^{\mathrm{e}}$ Melting temperatures and enthalpies were registered at a heating rate of $10^{\circ} \mathrm{C} \cdot \mathrm{min}^{-1}$. In parenthesis, values recorded in the second heating; ${ }^{\mathrm{f}}$ Crystallization temperatures and enthalpies were registered at cooling from $200{ }^{\circ} \mathrm{C}$ at $10^{\circ} \mathrm{C} \cdot \mathrm{min}^{-1}$; ${ }^{\mathrm{g}}$ Young's modulus measured at room temperature on a Zwick BZ2.5/TN1S; ${ }^{\mathrm{h}}$ Maximum tensile stress; ${ }^{\mathrm{i}}$ Maximum elongation at break.

All $\mathrm{PBS}_{x} \mathrm{SS}_{y}$ copolyesteres are semicrystalline with melting temperatures and enthalpies slightly decreasing with the content in SS units. Since the random insertion of the second comonomer will diminish the chain regularity of PBS, a decrease in both crystallite size and crystallinity should be expected for the copolyesters. Nevertheless the effect is really moderate with a decay of $T_{\mathrm{m}}$ in less than $10{ }^{\circ} \mathrm{C}$ for a content of $15 \mathrm{~mol} \%$ in SS units. The thermograms registered at the first heating from copolyester samples prepared by precipitation from $\mathrm{CHCl}_{3}$ with methanol are shown in Figure 3 . All of them display well-defined endothermic peaks characteristic of melting. Moreover, all of the copolyesters were able to crystallize from the melt upon cooling at $10^{\circ} \mathrm{C} \cdot \mathrm{min}^{-1}$ with a good reproduction of melting at the second heating (Figure S3). Samples obtained by precipitation from solution are 
expected to be well crystallized and to therefore display high melting temperature and enthalpy. Samples used for the second heating were crystallized under much more unfavorable conditions. The small differences observed in both $T_{\mathrm{m}}$ and $\Delta H_{\mathrm{m}}$ between first and second heating traces (Table 2) are indicative of the ability of these copolyesters to crystallize from the melt. In principle, it could be thought that ionic clusters that have not been destroyed after melting may act as nucleating agents favoring the crystallization of the molten material, as it was reported for these copolyesters with low content of SS units [22]. In the present case, however, the large supercooling observed for crystallization, which attains up to near $70^{\circ} \mathrm{C}$, makes such interpretation less probable.

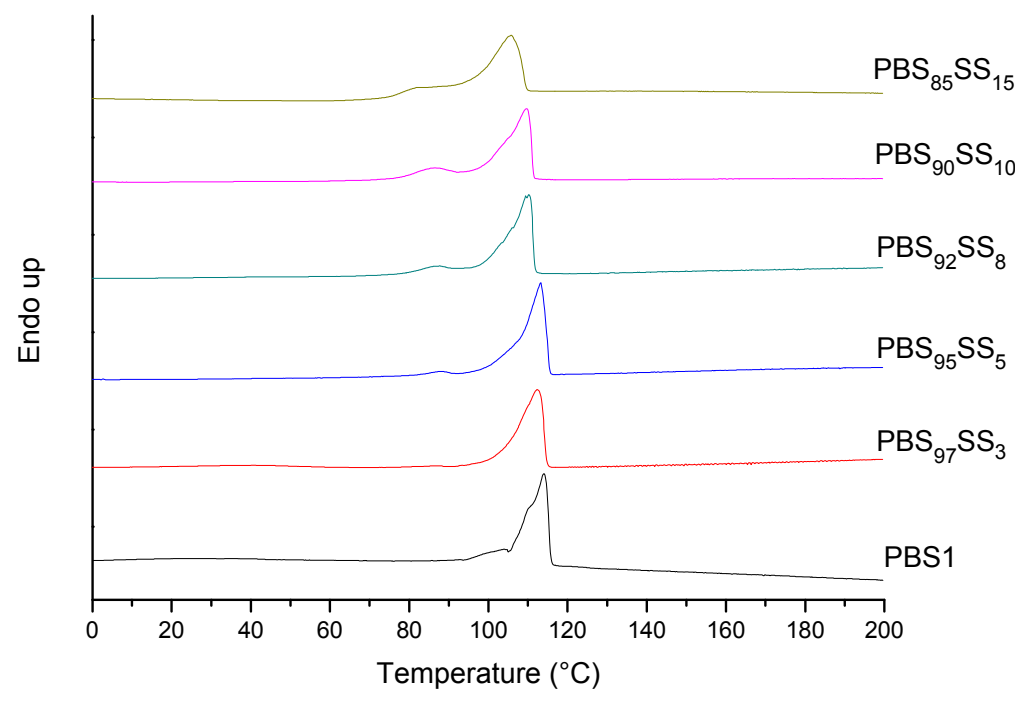

Figure 3. DSC thermograms (first heating) of $\mathrm{PBS}_{x} \mathrm{SS}_{y}$ copolyesters and $\mathrm{PBS} 1$.

The basic mechanical parameters of the copolyesters registered in tensile essays are presented in Table 2 together with those of PBS1 and PBS2 that have been included for comparison. The stress-strain curves experimentally obtained are depicted in Figure S4 of the Supplementary Informaiton file. Large differences in the mechanical parameters are observed between PBS1 and PBS2 according to what should be expected from their greatly different molecular weights. In fact, a drastic decay in extensibility in addition to slight reductions in the elastic modulus and maximum stress were observed for PBS when the molecular weight decreased from $\sim 100,000$ to $\sim 45,000$. Mechanical data for the $\mathrm{PBS}_{x} \mathrm{SS}_{y}$ series can be then analyzed as a function of composition by taking as reference PBS2, which has a molecular weight acceptably close to those of copolyesters. Sample PBS97 $\mathrm{SS}_{3}$ displays, however, an exceptional behavior due to its higher molecular weight and crystallinity; in this case, the three analyzed mechanical parameters are enhanced with respect to those of PBS2, as should be reasonably expected. The trend observed for the Young's modulus along the whole series is very clear: $E$ increased steadily with the content in SS units to reach near three-times the PBS value for a SS units content of about $15 \mathrm{~mol} \%$. Such a variation is in agreement with the increasing value of $T_{\mathrm{g}}$. The elongation at break was found to follow an opposite trend which is consistent with the variation observed for the elastic modulus. On the other hand, the influence of composition on the maximum stress is such that $\sigma_{\max }$ differences for copolyesters in the 5-10 mol\% range are less than $10 \%$ and may be considered just as due to uncontrolled experimental factors. The exceptional low value observed for $\mathrm{PBS}_{85} \mathrm{SS}_{15}$ can be explained by the lower crystallinity displayed by this sample. In spite of the lack of an apparent 
systematic variation in the measured parameters, it can be preliminarily concluded from this analysis that the insertion of ionic sulfonated units makes the polyester stiffer but also more brittle because of the high reduction undergone in the elongation to break. This behavior is in accordance with that displayed by other ionomers containing sulfonated units that have been reported by us in previous works [2,29].

\subsection{Hydrolytic Degradation}

Polyester recycling is in the focus of current polymer research concerning sustainability. Chemical recycling leading to monomer (or oligomer) recovery for subsequent valorization is an actual alternative method to physical processes, and PBS is a good candidate for hydrolysis recycling with regeneration of succinic acid [30]. Therefore, to know the influence of comonomers on novel PBS copolyesters is a matter of unquestionable importance.

Accordingly, the effect exerted by the incorporation of SS units on the hydrolytic degradation of PBS has been evaluated under a variety of conditions. The copolyesters chosen for comparison with PBS were $\mathrm{PBS}_{95} \mathrm{SS}_{5}$ and $\mathrm{PBS}_{85} \mathrm{SS}_{15}$. They were incubated in $\mathrm{pH} 7.4$ buffers at $37^{\circ} \mathrm{C}$ and also at $\mathrm{pH} 4.0$ and 10 to estimate their susceptibility to hydrolysis under both acidic and basic conditions. The degradation progress was monitored by following the weight loss and molecular weight decay of the residual sample as a function of the incubation time. These data are presented in Figure 4. After eight weeks of incubation at $\mathrm{pH} 7.4$, the $M_{\mathrm{w}}$ of samples with SS unit contents of 5 and $15 \mathrm{~mol} \%$ was $33 \%$ and $39 \%$ of the initial value, respectively whereas PBS decreased only in less than $20 \%$. It becomes clear, therefore, from these results that the hydrolytic degradation is enhanced by increasing contents in SS units.

To gain insight into the degradation of the polyester chain at the molecular level, PBS1, $\mathrm{PBS}_{95} \mathrm{SS}_{5}$ and $\mathrm{PBS}_{85} \mathrm{SS}_{15}$ polyesters were incubated at $37{ }^{\circ} \mathrm{C}$ in aqueous buffer at $\mathrm{pH} 4.0$ and $\mathrm{pH} 10$ and both the releasing fragments and the residual polymer were analyzed weekly over a period of two months. Changes taking place in sample weight and $M_{\mathrm{w}}$ of the three polyesters upon hydrolysis are comparatively depicted in Figure 4. A significant decay in both sample weight and molecular weight was observed for the $\mathrm{PBS}_{x} \mathrm{SS}_{y}$ copolyesters. It was observed that the hydrolytic degradation was drastically accelerated by increasing either ionic content or $\mathrm{pH}$. Similar observations have been recently reported for PBS ionenes [31] as well as for other copolyesters containing sulfoisophthalic units [2,18,29], and such increase has been attributed to the enhancement of the hydrophilicity caused by the presence of the ionic groups. 

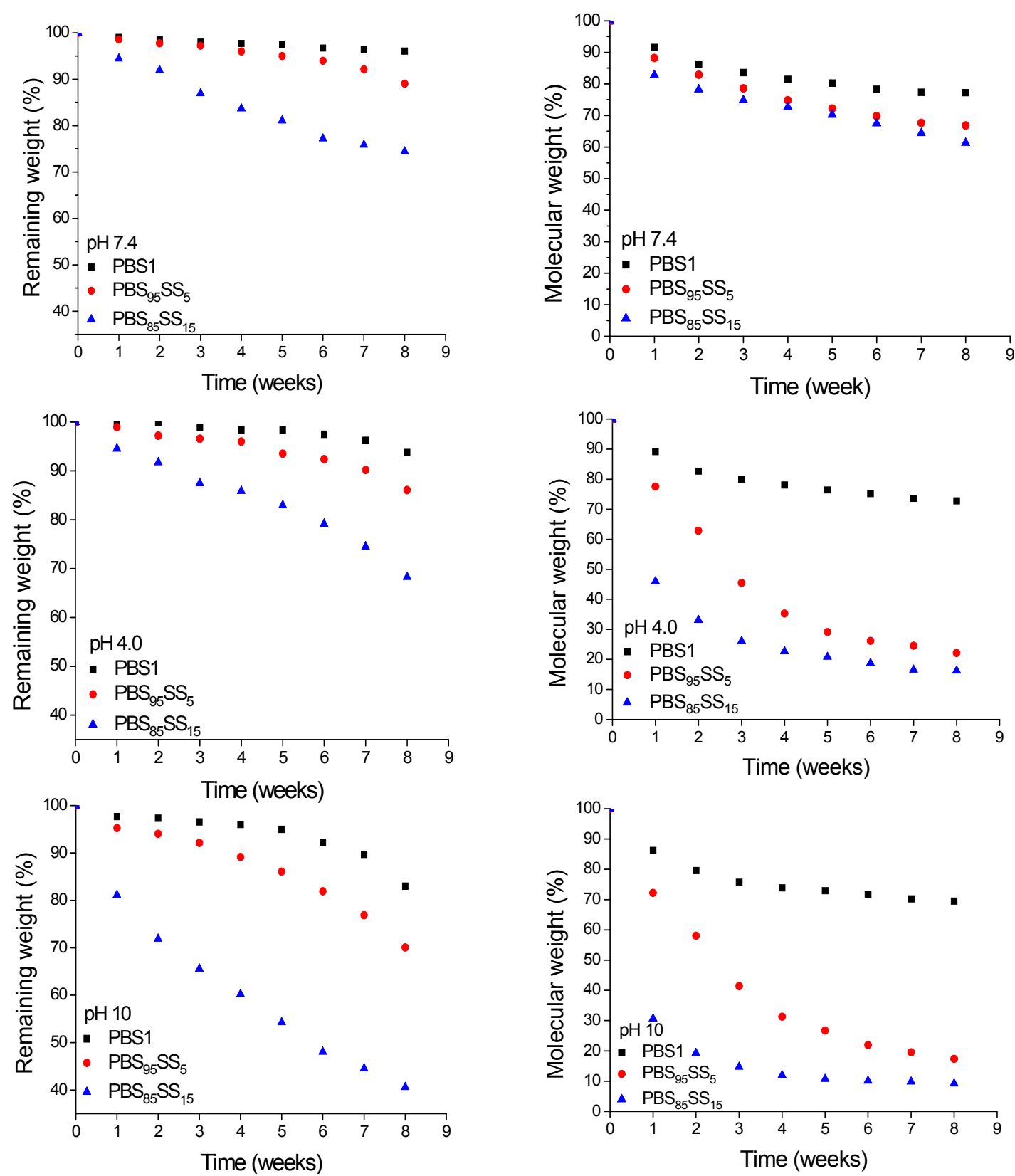

Figure 4. Evolution of sample remaining weight (left) and weight average molecular weight (right) for $\mathrm{PBS} 1, \mathrm{PBS}_{95} \mathrm{SS}_{5}$ and $\mathrm{PBS}_{85} \mathrm{SS}_{15}$ at $\mathrm{pH} 7.4,4.0$ and 10 (top to bottom) at $37^{\circ} \mathrm{C}$, as a function of incubation time.

Melting enthalpies displayed by $\mathrm{PBS} 1, \mathrm{PBSS}_{95} \mathrm{SS}_{5}$ and $\mathrm{PBS}_{85} \mathrm{SS}_{15}$ polyesters after incubation are compared in Figure 5. It is noteworthy to highlight that crystallinity of the three polyesters increased as degradation proceeded, with the highest values attained when samples were incubated in basic conditions. Such increase in crystallinity is indicative that hydrolysis has taken place preferably in the more permeable amorphous phase, as it is usually observed in semicrystalline polymers [32].

${ }^{1} \mathrm{H}$ NMR spectra of $\mathrm{PBS}_{85} \mathrm{SS}_{15}$ residue and of the products released to the incubation medium after eight weeks of incubation are depicted in Figure 6. It can be observed that the signals due to sulfonate units (ess and dss) almost disappeared in the degraded sample, and that signals arising from $-\mathrm{CH}_{2} \mathrm{OH}$ end groups increased as a consequence of the hydrolysis process. The spectra of water soluble products showed signals corresponding to succinic and sulfonated succinic acids, 1,4-butanediol and short 
oligomers, which are more intense for samples incubated at basic $\mathrm{pH}$ where copolyesters underwent a more severe degradation.

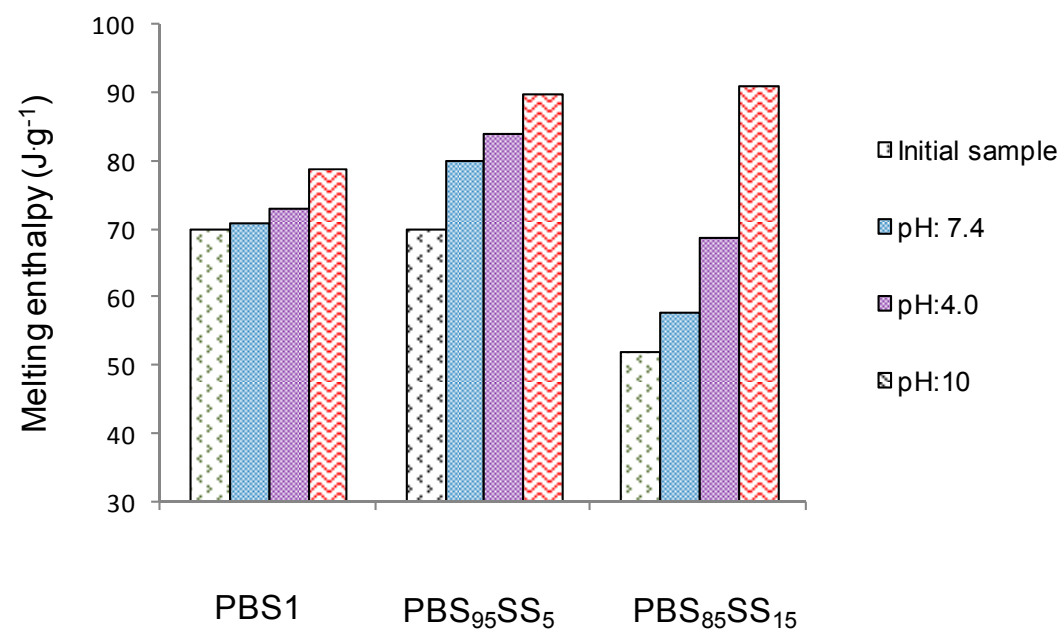

Figure 5. Melting enthalpies of $\mathrm{PBS} 1, \mathrm{PBS}_{95} \mathrm{SS}_{5}$ and $\mathrm{PBS}_{85} \mathrm{SS}_{15}$ after eight weeks of incubation and initial samples.

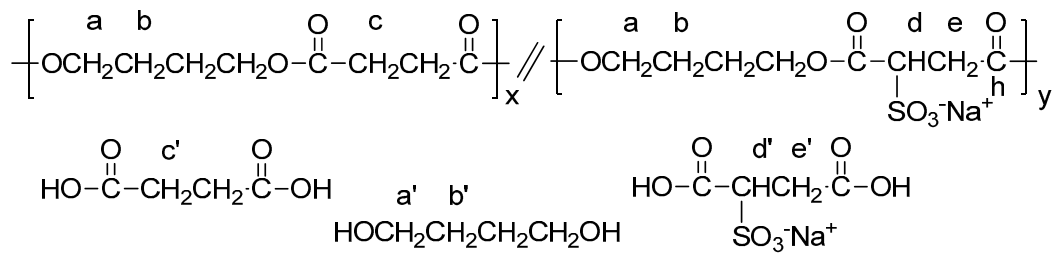

c)

HDO
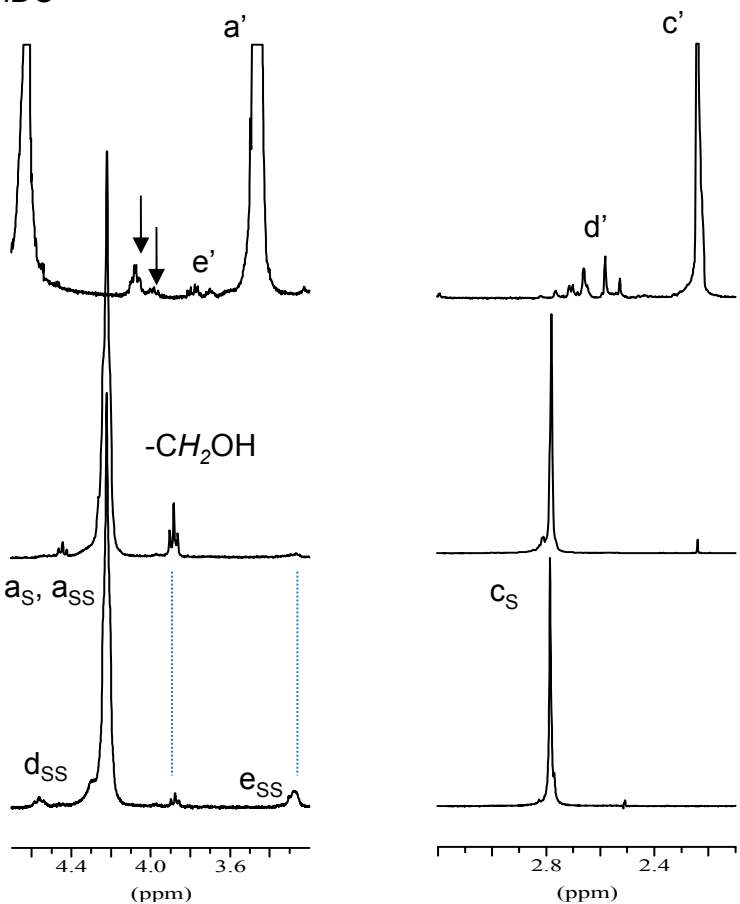

b'

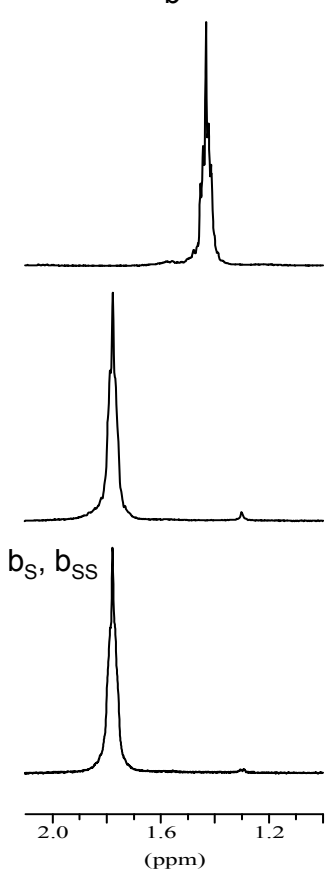

Figure 6. ${ }^{1} \mathrm{H}$ NMR spectra of $\mathrm{PBS}_{85} \mathrm{SS}_{15}$ (a) residue and (b) water soluble products (c) of $\mathrm{PBS}_{85} \mathrm{SS}_{15}$ degraded in aqueous buffer, $\mathrm{pH} 10$ for eight weeks. The arrows indicate signals arising from oligomers generated upon hydrolysis. 
The evolution of the content in sulfonated units of the residual $\mathrm{PBS}_{85} \mathrm{SS}_{15}$ with time for an incubation period of eight weeks under the different essayed conditions is plotted in Figure 7. These results are consistent with those obtained by sample weighing and GPC measurements and reveal that degradation involves preferably the hydrolysis of the SS units. The drastically accelerated degradation observed at $\mathrm{pH} 10$ is ascribed to both the higher hydrophilicity of the copolyester and to the faster and more efficient hydrolytic degradation process that takes place in copolyesters at basic $\mathrm{pH}$.

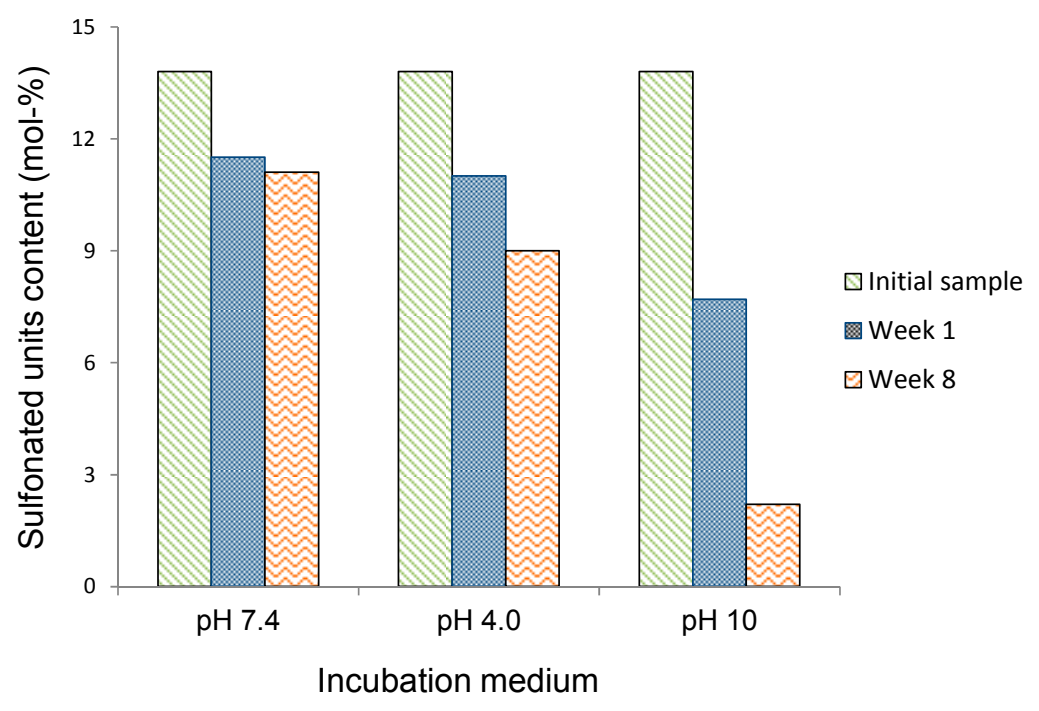

Figure 7. Evolution of the content in sulfonated units of the residual $\mathrm{PBS}_{85} \mathrm{SS}_{15}$ along incubation time.

\section{Conclusions}

High molecular weight PBS copolyesters containing amounts of ionic sulfonate groups up to $\sim 15$ mol\% were successfully synthesized by using sodium sulfonate dimethyl succinate as the comonomer. Polycondensation was carried out in the melt phase following essentially the same procedure that is used for the industrial preparation of PBS, although the reaction temperature had to be lower in order to prevent decomposition of the sulfonated compounds. The sulfonated diester effectively entered the growing polyester chain so that the compositions of the resulting copolyesters were quite close to those of the feed from which they were obtained. The weight-average molecular weights of the copolyesters were between 30,000 and 70,000 units, which is a range acceptable for most of their foreseeable applications. The changes in thermal properties of PBS due to copolymerization were not significant. The copolyesters continued to be thermally stable and semicrystalline, and they retained the ability to crystallize from the melt. Conversely, the mechanical behavior changed noticeably with both molecular weight and composition. In general, copolyesters were stiffer and stronger but also more brittle than PBS. The hydrodegradability was enhanced by copolymerization, an effect that was more pronounced when the copolyester was incubated in basic medium. All the property modifications were more apparent as the content in sulfonated units increased, and all the observed effects could be reasonably accounted for by the specific molecular interactions that are expected to occur from the presence of ionic units in the PBS chain. 


\section{Supplementary Information}

The ${ }^{1} \mathrm{H}$ and ${ }^{13} \mathrm{C}$ NMR spectra of SDMS monomer, the ${ }^{1} \mathrm{H}$ NMR spectrum of PBS $85 \mathrm{SS}_{15}$ copolyester after heating at $210{ }^{\circ} \mathrm{C}$, the second heating DSC thermograms and the strain-stress curves of $\mathrm{PBS}_{x} \mathrm{SS}_{y}$ copolyesters can be accessed at http://www.mdpi.com/2073-4360/7/7/1232/s1.

\section{Acknowledgments}

Financial support for this work was provided by MINECO (Spain) with Grant MAT-2012-38044CO3-03 and by AGAUR (Catalonia) with grant 2009SGR1469. We would also like to thank Universitat Politècnica de Catalunya for the UPC-Research grant awarded to Mayka Bautista.

\section{Author Contributions}

Mayka Bautista is the Ph.D. student that took charge of most of the experimental work. Abdel Alla performed the DSC and TGA analysis and assisted with the molecular weights determinations. Antxon Martínez de Ilarduya was responsible for the NMR analyses and collaborated with Sebastián Muñoz-Guerra to organize and supervise the work and to write this paper.

\section{Conflicts of Interest}

The authors declare no conflict of interest.

\section{References}

1. Eisenberg, A.; Rinaudo, M. Polyelectrolites and ionomers. Polym. Bull. 1990, 24, 671-671.

2. Bautista, M.; Martínez de Ilarduya, A.; Alla, A.; Muñoz-Guerra, S. Sulfonated poly(hexamethylene terephthalate) copolyesters: Enhanced thermal and mechanical properties. J. Appl. Polym. Sci. 2013, 129, 3527-3535.

3. Lu, X.Y.; Weiss, R.A. Morphology and phase-behavior of blends of a styrenic block-copolymer ionomer and poly(caprolactone). Macromolecules 1993, 26, 3615-3622.

4. Kim, J.S.; Hong, M.C.; Nah, Y.H. Effects of two ionic groups in an ionic repeat unit on the properties of styrene ionomers. Macromolecules 2002, 35, 155-160.

5. Szymczyk, A.; Roslaniec, Z. Sulfonated poly(ether-block-ester) ionomers with anions in the polyester hard segments. Polym. Adv. Technol. 1999, 10, 579-587.

6. Nguyen, D.; Kim, J.S.; Guiver, M.D.; Eisenberg, A. Clustering in carboxylated polysulfone ionomers: A characterization by dynamic mechanical and small-angle X-ray scattering methods. J. Polym. Sci. B 1999, 37, 3226-3232.

7. Eisenberg, A.; Hird, B.; Moore, R.B. A new multiplet-cluster model for the morphology of random ionomers. Macromolecules 1990, 23, 4098-4107.

8. Eisenberg, A. Clustering of ions in organic polymers. A theoretical approach. Macromolecules 1970, 3, 147-154.

9. Han, S.I.; Yoo, Y.; Kim, D.K.; Im, S.S. Biodegradable aliphatic polyester ionomers. Macromol. Biosci. 2004, 4, 199-207. 
10. Stevens, E.S. Green Plastics: An Introduction to the New Science of Biodegradable Plastics. Princeton University Press: Princeton, NJ, USA, 2002.

11. Scherer, T.M.; Fuller, R.C.; Lenz, R.W.; Goodwin, S. Hydrolase activity of an extracellular depolymerase from Aspergillus fumigatus with bacterial and synthetic polyesters. Polym. Degrad. Stab. 1999, 64, 267-275.

12. Edlund, U.; Albertsson, A.C. Polyesters based on diacid monomers. Adv. Drug Deliv. Rev. 2003, 55, 585-609.

13. Penco, M.; Sartore, L.; Bignotti, F.; D’Antone, S.; di Landro, L. Thermal properties of a new class of block copolymers based on segments of poly(D,L-lactic-glycolic acid) and poly(epsilon-caprolactone). Eur. Polym. J. 2000, 36, 901-908.

14. Park, J.W.; Kim, D.K.; Im, S.S. Crystallization behaviour of poly(butylene succinate) copolymers. Polym. Int. 2002, 51, 239-244.

15. Abe, H.; Doi, Y.; Hori, Y.; Hagiwara, T. Physical properties and enzymatic degradability of copolymers of $(R)$-3-hydroxybutyric acid and $(S, S)$-lactide. Polymer 1998, 39, 59-67.

16. Howard, G.J.; Knutton, S. Isomorphism in aliphatic copolyesters. Polymer 1968, 9, 527-534.

17. Schulken, R.M.; Boy, R.E.; Cox, R.H. Differential thermal analysis of linear polyesters. J. Polym. Sci. C 1964, 6, 17-25.

18. Bougarech, A.; Abid, M.; Gouanve, E.; Espuche, E.; Abid, S.; El Gharbi, R.; Fleury, E. Synthesis, characterization and water sorption study of new biobased (furanic-sulfonated) copolyesters. Polymer 2013, 54, 5482-5489.

19. Bougarech, A.; Abid, M.; DaCruz-Boisson, F.; Abid, S.; El Gharbi, R.; Fleury, E. Modulation of furanic-sulfonated isophthalic copolyesters properties through diols units control. Eur. Polym. J. 2014, 58, 207-217.

20. Han, S.I.; Im, S.S.; Kim, D.K. Dynamic mechanical and melt rheological properties of sulfonated poly(butylene succinate) ionomers. Polymer 2003, 44, 7165-7173.

21. Park, S.B.; Hwang, S.Y.; Moon, C.W.; Im, S.S.; Yoo, E.S. Plasticizer effect of novel PBS ionomer in PLA/PBS ionomer blends. Macromol. Res. 2010, 18, 463-471.

22. Ishida, K.; Han, S.I.; Inoue, Y.; Im, S.S. Novel poly(butylene succinate)-based ionomers with sulfonated succinate units: Synthesis, morphology, and the unique nucleation effect on crystallization. Macromol. Chem. Phys. 2005, 206, 1028-1034.

23. Lim, J.S.; Lee, Y.; Im, S.S. Influence of ionic association on the nonisothermal crystallization kinetics of sodium sulfonate poly(butylene succinate) ionomers. J. Polym. Sci. B 2008, 46, 925-937.

24. Wang, X.L.; Wang, L.; Li, H.; Tang, X.Z.; Chang, F.C. Syntheses of poly(ethylene oxide) polyurethane ionomers. J. Appl. Polym. Sci. 2000, 77, 184-188.

25. Greener, J.; Gillmor, J.R.; Daly, R.C. Melt rheology of a class of polyester ionomers. Macromolecules 1993, 26, 6416-6424.

26. Kang, H.Y.; Long, T.E. Synthesis and characterization of telechelic sulfonate polyester ionomers. Abstr. Papers Am. Chem. Soc. 2001, 221, U435-U435.

27. Eisenberg, A.; Navratil, M. Ion clustering and viscoelastic relaxation in styrene-based inomers. 2. Effect of ion concentration. Macromolecules 1973, 6, 604-612.

28. Shohamy, E.; Eisenberg, A. Effect of ion aggregation on some melt properties of styrene ionomers. J. Polym. Sci. B Polym. Phys. 1976, 14, 1211-1220. 
29. Gaona, O.; Kint, D.; Martinez de Ilarduya, A.; Alla, A.; Bou, J.; Muñoz-Guerra, S. Preparation and hydrolytic degradation of sulfonated poly(ethylene terephthalate) copolymers. Polymer 2003, 44, 7281-7289.

30. Kanemura, C.; Nakashima, S.; Hotta, A. Mechanical properties and chemical structures of biodegradable poly(butylene-succinate) for material reprocessing. Polym. Degrad. Stab. 2012, 97, 972-980.

31. Wu, F.; Huang, C.L.; Zeng, J.B.; Li, S.L.; Wang, Y.Z. Synthesis and characterization of segmented poly(butylene succinate) urethane ionenes containing secondary amine cation. Polymer 2014, 55, 4358-4368.

32. Cho, K.; Lee, J.; Kwon, K. Hydrolytic degradation behavior of poly(butylene succinate)s with different crystalline morphologies. J. Appl. Polym. Sci. 2001, 79, 1025-1033.

(C) 2015 by the authors; licensee MDPI, Basel, Switzerland. This article is an open access article distributed under the terms and conditions of the Creative Commons Attribution license (http://creativecommons.org/licenses/by/4.0/). 